\title{
Enhanced Optical Absorption Due to Symmetry Breaking in $\mathrm{TiO}_{2(1-x)} \mathrm{S}_{2 x}$ Alloys
}

\author{
André Schleife, ${ }^{\dagger, \ddagger}$ Patrick Rinke, ${ }^{\S, \|, \ddagger}$ Friedhelm Bechstedt, ${ }^{\perp, \ddagger}$ and Chris G. Van de Walle ${ }^{*, \|}$ \\ ${ }^{\dagger}$ Condensed Matter and Materials Division, Lawrence Livermore National Laboratory, Livermore, California 94550, United States \\ ${ }^{\ddagger}$ European Theoretical Spectroscopy Facility (ETSF) \\ ${ }^{\S}$ Fritz-Haber-Institut der Max-Planck-Gesellschaft, Faradayweg 4-6, 14195 Berlin, Germany \\ "Materials Department, University of California, Santa Barbara, California 93106-5050, United States \\ ${ }^{\perp}$ Institut für Festkörpertheorie und -optik, Friedrich-Schiller-Universität, Max-Wien-Platz 1, 07743 Jena, Germany
}

ABSTRACT: Titania $\left(\mathrm{TiO}_{2}\right)$ is frequently used in photovoltaic and photocatalytic applications, despite the fact that its main optical absorption occurs only at $\sim 4 \mathrm{eV}$. Absorption across the band gap of $3 \mathrm{eV}$ is dipole-forbidden in rutile $\mathrm{TiO}_{2}$. By means of first-principles theoretical spectroscopy calculations, we demonstrate that alloying with $\mathrm{TiS}_{2}$ introduces an absorption band into the fundamental gap of $\mathrm{TiO}_{2}$. In addition, band-edge transitions contribute to optical absorption because the $\mathrm{S}$ incorporation breaks the symmetry of the $\mathrm{TiO}_{2}$ lattice. Both effects lead to pronounced absorption of visible light for $\mathrm{S}$ concentrations as low as $1.5 \%$.

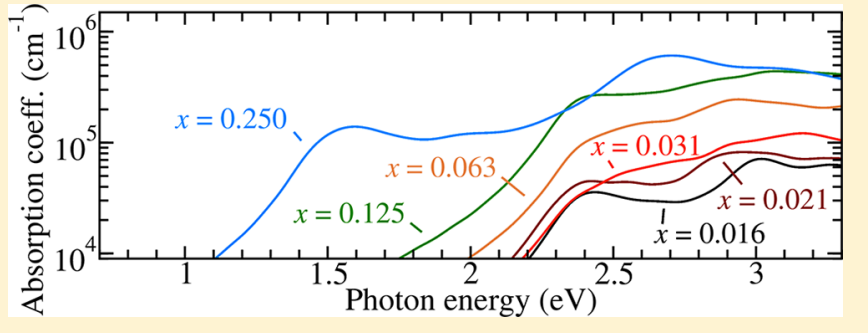

increase in absorption in the visible range for $S$ concentrations as low as $1.5 \%$. Our calculations also explain why experimental studies of $S$ incorporation observed a corresponding red shift of the absorption onset ${ }^{15,16,22-24}$ and not the emergence of a defect-related peak in the band gap region.

\section{METHODOLOGY}

We model $\mathrm{TiO}_{2(1-x)} \mathrm{S}_{2 x}$ alloys of different compositions $x$ ranging from 0 to 0.25 by replacing one $\mathrm{O}$ atom with one $\mathrm{S}$ atom in rutile supercells of 12 to 96 atoms. The geometries were fully relaxed in density functional theory. Our study focuses on rutile $\mathrm{TiO}_{2}$ (space-group: $P 4_{2} / \mathrm{mnm}$ or $D_{4 h}^{14}$ (SG136)), because we want to study the optical-absorption properties for the band structures reported in ref 17 to investigate the impact of the dipole-selection rules. Nevertheless, the symmetry-breaking and lattice-distortion mechanism discussed below is expected to also apply to the anatase phase because it is driven by the different radii of the oxygen and the sulfur atoms. Optical spectra are calculated using manybody perturbation theory by solving the Bethe-Salpeter equation (BSE). ${ }^{25}$

In a first step, the Kohn-Sham ${ }^{26}$ (KS) eigenvalues and eigenstates are calculated using the local-density approximation $(\mathrm{LDA})^{27,28}$ to exchange and correlation. The projectoraugmented wave method ${ }^{29}$ as implemented in the Vienna ab initio simulation package $e^{30,31}$ is used to describe the electronion interaction. Pseudopotentials are the same as those used in

Received: October 29, 2012

Revised: January 18, 2013

Published: January 28, 2013 


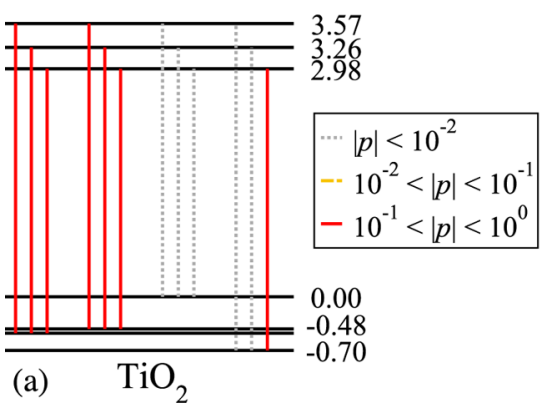

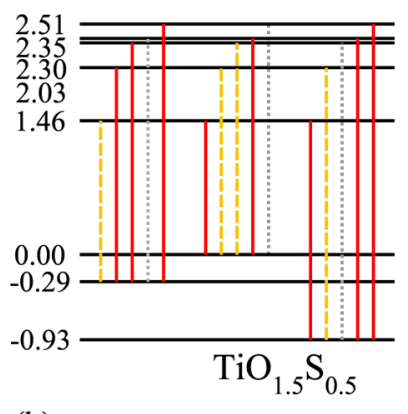

(b)

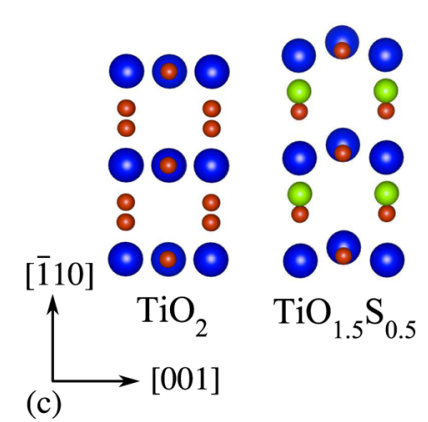

Figure 1. Absolute values $|p|\left(\right.$ in $\hbar / a_{\mathrm{B}}$ ) of the optical transition matrix elements for $\mathrm{TiO}_{2}$ (a) and the alloy with $x=0.25$ (b). The energy zero in both spectra is the VBM. The alloy spectrum has been shifted such that the state that derives from the $\mathrm{TiO}_{2} \mathrm{VBM}$ is aligned with the $\mathrm{VBM}$ in bulk $\mathrm{TiO}$. In panel $\mathrm{c}$, we visualize the atomic geometries for these two cases using blue (big) circles for $\mathrm{Ti}$ atoms, red (small) circles for $\mathrm{O}$ atoms, and green (intermediate) circles for $\mathrm{S}$ atoms.

ref 17 . From the KS wave functions, the optical transitionmatrix elements are calculated using the longitudinal approximation. $^{32}$

Excitonic effects as well as local-field effects play a dominant role in the absorption spectra of oxides ${ }^{33}$ and their alloys. ${ }^{34}$ The description of these effects goes beyond the KS picture and requires the framework of many-body perturbation theory by solving the BSE for the optical polarization function $P .{ }^{25}$ In the optical limit of vanishing photon wave vector, the frequencydependent macroscopic dielectric tensor $\varepsilon_{i j^{\prime}}(\omega)$ (with $j, j^{\prime}=$ $\{x, y, z\})$ follows directly from $P .^{25}$ All linear optical properties of a material can then be derived from $\varepsilon_{i j^{\prime}}(\omega)$; specifically, the frequency-dependent optical absorption coefficient $\alpha_{j}(\omega)$

$$
\alpha_{j}(\omega)=\frac{\sqrt{2} \omega}{c} \sqrt{\left|\varepsilon_{j j}(\omega)\right|-\operatorname{Re} \varepsilon_{j j}(\omega)}
$$

To make the solution of the BSE tractable, it is typically rewritten as an eigenvalue problem for the excitonic electronhole pair Hamiltonian. ${ }^{25}$ In our BSE code ${ }^{35}$ the KS eigenstates are used to compute the matrix elements of the statically screened Coulomb attraction between electrons and holes as well as the unscreened exchange terms, both of which determine the excitonic Hamiltonian. In addition, the electron and hole quasiparticle energies are required. Whereas it is desirable to calculate these within many-body perturbation theory in Hedin's $G W$ approach, ${ }^{36}$ this task is computationally infeasible at the moment, especially for unit cells containing as many as 96 atoms and k-point meshes as dense as the ones used in this work (see below). Test $G_{0} W_{0}$ calculations based on LDA input for the 12 -atom cell $(x=0.25)$ reveal that the position of the S-induced defect level in the band gap agrees to within $0.05 \mathrm{eV}$ with the scissor-corrected band structure of ref 17 in which the occupied bands are rigidly shifted to higher energies. Because the conclusions of the present work are not affected by such a relatively small error, we apply these rigid shifts to all supercells in this work. The resulting gap of $\mathrm{TiO}_{2}$ amounts to $2.98 \mathrm{eV} .^{17}$

Converging the optical absorption spectrum in the vicinity of the band edge requires a large number of $k$ points. ${ }^{37}$ We use different (hybrid ${ }^{37}$ ) k-point meshes for the different supercells to sample the low-energy transitions, with a maximum k-point distance of $0.36 \AA^{-1}$ in all cases. In addition, we reduce the $\mathbf{k}$ sampling when increasing the number of conduction bands for the high-energy transitions. A direct diagonalization of the resulting excitonic Hamiltonian matrices quickly becomes infeasible as ranks reach up to 100000 . In this work, we therefore employ an efficient time-evolution scheme ${ }^{38}$ to calculate $\varepsilon(\omega)$ from the excitonic Hamiltonian. Optical transitions with transition energies larger than the BSE cutoff are included up to $200 \mathrm{eV}$ on the KS level (as described in ref $39)$ to obtain converged results for the real part of $\varepsilon(\omega)$ at low photon energies.

\section{RESULTS AND DISCUSSION}

We first illustrate the principal effects of $S$ incorporation for the highest concentration. Figure 1 displays the energies of the lowest conduction and highest valence states at the $\Gamma$ point in $\mathrm{TiO}_{2}$ and in the alloy with $x=0.25$, together with the dipole transition matrix elements and the corresponding distortion of the atomic geometries. The lowest energy transition is dipoleforbidden in $\mathrm{TiO}_{2}$ independent of the light polarization, and optical transitions across the fundamental gap therefore do not contribute to the onset of absorption. The first transitions with any appreciable weight at the $\Gamma$ point originate not from the VBM but from the two bands $\sim 0.5 \mathrm{eV}$ lower in energy. These transitions build up to the first strong absorption peak in $\mathrm{TiO}_{2}$ at $\sim 4.0 \mathrm{eV}$.

Replacing one oxygen atom in the rutile unit cell by a sulfur atom introduces $\mathrm{S}$-derived levels in the fundamental gap of $\mathrm{TiO}_{2}$, as seen in Figure 1b. The dipole matrix elements for transitions between these states and the $\mathrm{TiO}_{2}$-derived conduction bands are large and contribute considerably to optical absorption in the visible spectral range. However, the $S$ atom also perturbs the rutile crystal structure, as can be seen in Figure 1c. This leads to $\mathrm{Ti}$ atoms moving out-of-plane, which, in addition to replacing an oxygen atom by a sulfur atom, further breaks the rutile symmetry of the lattice. The distance between $S$ and the next-nearest $\mathrm{Ti}$ atom amounts to $\sim 2.25 \AA$, whereas the $\mathrm{Ti}-\mathrm{O}$ distance is only $\sim 1.90 \AA$. A combination of this lattice distortion and the hybridization with $S$ states facilitates absorption from the states that derive from the band edges in $\mathrm{TiO}_{2}$. This effect adds absorption in the nearultraviolet spectral region that is absent in pure $\mathrm{TiO}_{2}$ and applies to all $S$ concentrations we have investigated with a strength proportional to the $S$ concentration.

In Figure 2, we investigate the band structure of $\mathrm{TiO}_{2(1-x)} \mathrm{S}_{2 x}$ with $x=0.015625$, the lowest $S$ concentration we have considered. The alloy exhibits an isolated defect-induced band at $\sim 0.6 \mathrm{eV}$ above the bulk VBM of $\mathrm{TiO}_{2}$. This band possesses strong $S 3 p$ character, similar to the $\mathrm{O} 2 \mathrm{p}$ symmetry of the VBM. Because of the perturbation by the $S$ atom, the gap 
(a)

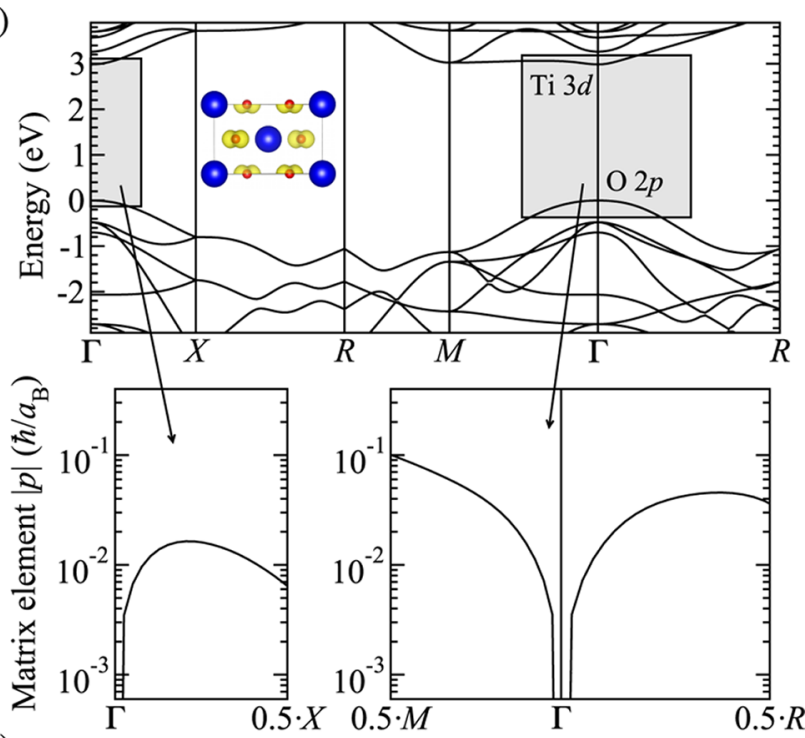

(b)

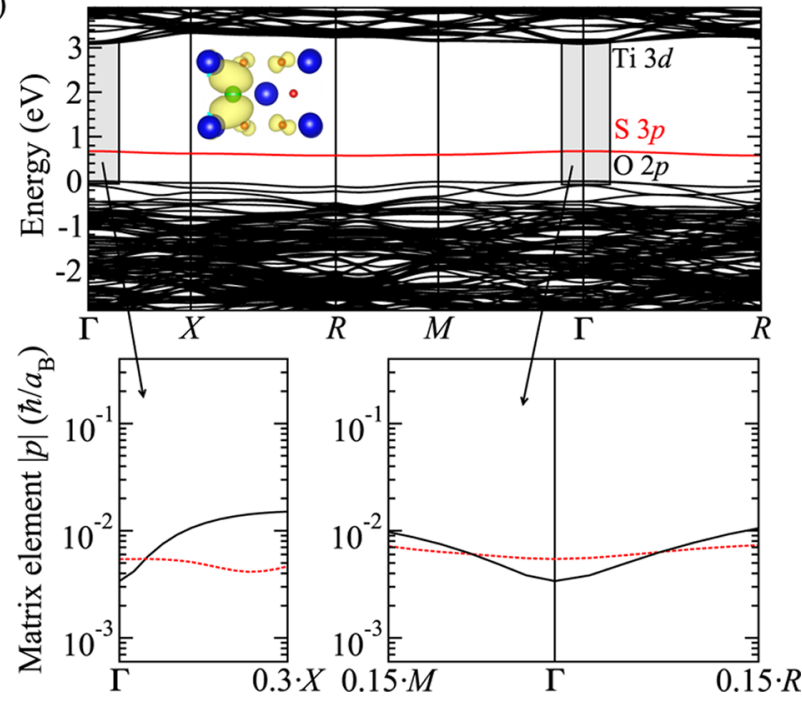

Figure 2. Scissor-corrected single-particle band structures of $\mathrm{TiO}_{2(1-x)} \mathrm{S}_{2 x}$ and optical matrix elements for (a) $x=0$ and (b) $x=$ 0.015625 . The defect-induced level is shown in red and the VBM of bulk $\mathrm{TiO}_{2}$ has been used as energy zero. The optical transition matrix elements $1 / 2\left(\left|p_{x}\right|+\left|p_{y}\right|\right)$ are plotted for transitions originating from the uppermost bulk-like valence band (black lines) or the defectinduced level (dashed red lines) into the lowest conduction band for the $k$-space regions indicated by the shaded boxes. $\left|p_{z}\right|$ is too small to be included in the plot. Isosurfaces for the partial charge density of the uppermost valence band are shown in the insets.

between the bulk-derived valence- and conduction-band states increases slightly to $3.08 \mathrm{eV}$.

Figure 2 also depicts the absolute values of the optical transition matrix elements for electronic excitations from the bulk-like valence and the $S$-induced levels into the conduction bands. In pure $\mathrm{TiO}_{2}$, the dipole matrix element vanishes directly at the $\Gamma$-point, although the value rises sharply off $\Gamma$, as previously observed in $\mathrm{SnO}_{2} \cdot{ }^{33}$ Sulfur incorporation breaks the rutile crystal symmetry and the transition becomes allowed. Note that even for compositions as low as $1.5 \%$ the perturbation of the lattice symmetry is still strong enough to render transitions from the valence-band edge (that are dipoleforbidden in pure $\mathrm{TiO}_{2}$ ) as large as the one from the S-induced state. The isosurfaces of the partial charge density of the respective uppermost valence band in the insets of Figure 2 clearly show the hybridization of $\mathrm{O}$ - and S-derived states and also that these states are less localized in the alloy system.

To support this qualitative discussion, we give quantitative optical spectra based on the imaginary part of the dielectric function in Figure 3 for different alloy compositions $x$. As

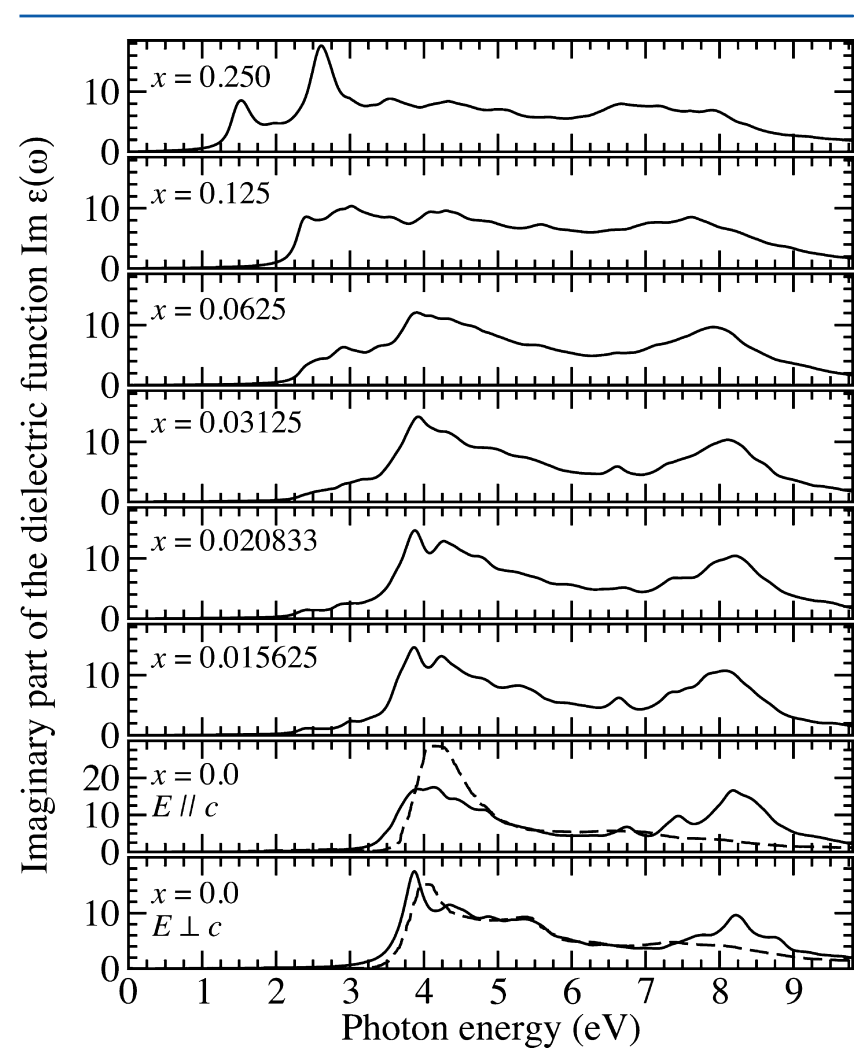

Figure 3. Imaginary part of the dielectric function (averaged over all polarization directions) versus incident photon energy (in electronvolts) for different $\mathrm{TiO}_{2(1-x)} \mathrm{S}_{2 x}$ alloy compositions. A Lorentzian broadening of $0.1 \mathrm{eV}$ is used to account for temperature and lifetime effects. For bulk $\mathrm{TiO}_{2}$, we compare the calculated dielectric function to the measurements of ref 7 (dashed lines) for two polarization directions.

previously mentioned, the first main peak occurs around $4 \mathrm{eV}$ in pure $\mathrm{TiO}_{2}$ and originates from lower lying valence bands. Another peak appears around $8.2 \mathrm{eV}$. The position and the spectral shape of the onset as well as of this second peak agree well with other theoretical results obtained within the BetheSalpeter framework. ${ }^{8-11}$ Our results also agree with the experimental spectra by Cardona and Harbeke, ${ }^{7}$ also included in Figure 3. The deviation of the energy position of the absorption onset is small; also, the curve shape agrees well, in particular for the case of perpendicular light polarization, confirming that our procedure of rigidly shifting unoccupied states is justified.

Increasing the $S$ content in the alloy leads to a considerable reduction of the energy position of the absorption onset. For concentrations as low as $1.5 \%$, the onset has already moved to $\sim 2.4 \mathrm{eV}$, that is, into the visible spectral range. The absorption below $3 \mathrm{eV}$ can be traced to transitions of electrons from the $\mathrm{S}$ level into the lowest conduction-band states. However, the small peak around $3 \mathrm{eV}$ for $1.5 \%$ originates from transitions between the bulk-like VBM and the conduction-band minimum that have become dipole allowed. In addition, this peak 
increases in strength and shifts to smaller energies as the composition $x$ of the alloy increases; it occurs around $2.5 \mathrm{eV}$ for $x=0.25$. (See also Figure 1.)

Increasing $x$ leads to new peak structures in the imaginary part of the dielectric function, especially in the energy range between 2.4 and $3.9 \mathrm{eV}$. (See Figure 3.) Spectral weight is shifted from the peak at around $3.9 \mathrm{eV}$ to lower photon energies. This peak, which is very pronounced for pure $\mathrm{TiO}_{2}$, becomes increasingly broad with increasing $x$. We find significant absorption due to transitions from levels with strong $S$ character (cf. Figure 3) already for $x \geq 0.0625$ and these contributions evolve into a peak in the absorption spectrum at $\sim 1.5 \mathrm{eV}$ for $x=0.25$.

For a direct comparison to experimentally reported absorption coefficients, we use our calculated dielectric functions to compute $\alpha(\omega)$ using eq 1 ; the results are shown in Figure 4. The main effect of S incorporation is to introduce a

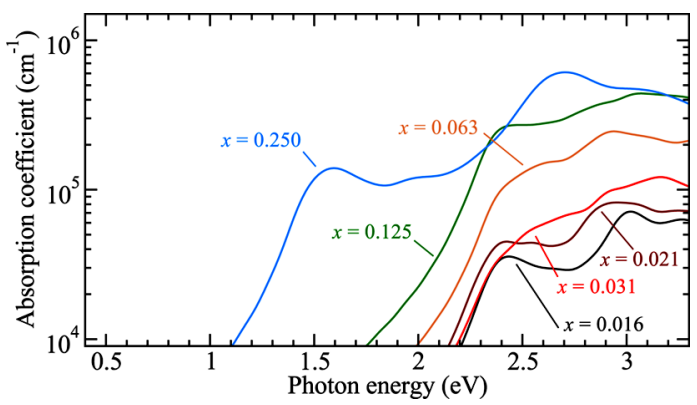

Figure 4. Optical absorption coefficient (averaged over all polarization directions) of $\mathrm{TiO}_{2(1-x)} \mathrm{S}_{2 x}$ versus incident photon energy (in electronvolts) for different alloy compositions. A Lorentzian broadening of $0.1 \mathrm{eV}$ is used to simulate temperature and lifetime effects; the resulting sub-band gap absorption in $\mathrm{TiO}_{2}$, which is smaller than $2 \times$ $10^{4} \mathrm{~cm}^{-1}$ below $2.8 \mathrm{eV}$, has been subtracted from all the curves.

red shift of the absorption onset, not a defect-related peak in the band gap region; as discussed above, the absorption related directly to the $S$-induced states is small in magnitude until the $S$ concentration becomes large (approaching $x=0.25$ ). The red shift of the absorption onset is considerable even for concentrations as low as $1.5 \%$ and gives rise to notable absorption in the visible spectral region. For larger $S$ concentrations, the absorption edge is further red-shifted, and the magnitude of absorption in the visible spectral region increases. Whereas this is consistent with experimental observations, ${ }^{15,16,22-24}$ our results explicitly show the importance of the symmetry breaking for explaining the observed absorption. This symmetry breaking mechanism should not strongly depend on the nature of the dopant; hence, we expect an impact also for other dopants, which is consistent with experimental studies using, for instance, nitrogen. ${ }^{15,16}$

\section{CONCLUSIONS}

In summary, we have presented state-of-the-art theoretical spectroscopy calculations for the optical properties of $\mathrm{TiO}_{2(1-x)} \mathrm{S}_{2 x}$ alloys. $\mathrm{S}$ incorporation significantly reduces the energy position of the optical absorption onset compared with pure $\mathrm{TiO}_{2}$. We ascribe this red shift in part to absorption from a $S$-induced band in the band gap and in part to band-edge absorption that becomes dipole-allowed in the alloy due to the broken crystal symmetry. Only at fairly large $S$ concentrations does the absorption from the S-induced band become dominant.

We found the symmetry breaking to be a reason for a significant contribution to optical absorption. Because this mechanism is universal and does not depend on the nature of the dopant, we expect this principle to be transferable to other materials whose band-edge transitions are dipole-forbidden, such as $\mathrm{SnO}_{2}$ or $\mathrm{In}_{2} \mathrm{O}_{3}$ : the incorporation of chalcogenide atoms on the oxygen sublattice should have similar effects as in the case of $\mathrm{TiO}_{2}$.

\section{AUTHOR INFORMATION}

\section{Corresponding Author}

*Phone: +1 805 8937144. E-mail: vandewalle@mrl.ucsb.edu.

\section{Notes}

The authors declare no competing financial interest.

\section{ACKNOWLEDGMENTS}

We acknowledge fruitful discussions with A. Janotti and C. Rödl. A.S., P.R., and F.B. gratefully acknowledge financial support by the European Community within the e-I3 project ETSF (GA no. 211956) and the Deutsche Forschungsgemeinschaft (FB: project No. Be 1346/20-1, PR: project no. Ri 1507/ 3-1). CVdW was supported as part of the Center for Energy Efficient Materials, an Energy Frontier Research Center funded by the U.S. DOE, BES under award number DE-SC0001009. Part of this work was performed under the auspices of the U.S. Department of Energy at Lawrence Livermore National Laboratory under contract DE-AC52-07A27344. P.R. and F.B. acknowledge the NSF IMI Program (DMR-0843934).

\section{REFERENCES}

(1) Grant, F. A. Properties of Rutile (Titanium Dioxide). Rev. Mod. Phys. 1959, 31, 646-674.

(2) Diebold, U. The Surface Science of Titanium Dioxide. Surf. Sci. Rep. 2003, 48, 53-229.

(3) O’Regan, B.; Grätzel, M. A. Low-Cost, High-Efficiency Solar Cell Based on Dye-Sensitized Colloidal $\mathrm{TiO}_{2}$ Films. Nature 1991, 353, 737-740.

(4) Fujishima, A.; Honda, K. Electrochemical Photolysis of Water at a Semiconductor Electrode. Nature 1972, 238, 37-38.

(5) Hoffmann, M. R.; Martin, S. T.; Choi, W.; Bahnemann, D. W. Environmental Applications of Semiconductor Photocatalysis. Chem. Rev. 1995, 95, 69-96.

(6) Pascual, J.; Camassel, J.; Mathieu, H. Fine Structure in the Intrinsic Absorption Edge of $\mathrm{TiO}_{2}$. Phys. Rev. B 1978, 18, 5606-5614.

(7) Cardona, M.; Harbeke, G. Optical Properties and Band Structure of Wurtzite-Type Crystals and Rutile. Phys. Rev. 1965, 137, A1467A1476.

(8) Kang, W.; Hybertsen, M. S. Quasiparticle and Optical Properties of Rutile and Anatase $\mathrm{TiO}_{2}$. Phys. Rev. B 2010, 82, 085203/1-085203/ 11.

(9) Lawler, H. M.; Rehr, J. J.; Vila, F.; Dalosto, S. D.; Shirley, E. L.; Levine, Z. H. Optical to UV Spectra and Birefringence of $\mathrm{SiO}_{2}$ and $\mathrm{TiO}_{2}$ : First-Principles Calculations with Excitonic Effects. Phys. Rev. B 2008, 78, 205108/1-205108/8

(10) Chiodo, L.; García-Lastra, J. M.; Iacomino, A.; Ossicini, S.; Zhao, J.; Petek, H.; Rubio, A. Self-Energy and Excitonic Effects in the Electronic and Optical Properties of $\mathrm{TiO}_{2}$ Crystalline Phases. Phys. Rev. B 2010, 82, 045207/1-045207/12.

(11) Landmann, M.; Rauls, E.; Schmidt, W. G. The Electronic Structure and Optical Response of Rutile, Anatase and Brookite $\mathrm{TiO}_{2}$. J. Phys.: Condens. Mater. 2012, 24, 195503/1-195503/6. 
(12) Chen, D.; Xu, G.; Miao, L.; Chen, L.; Nakao, S.; Jin, P. WDoped Anatase $\mathrm{TiO}_{2}$ Transparent Conductive Oxide Films: Theory and Experiment. J. Appl. Phys. 2010, 107, 063707/1-063707/4.

(13) Umebayashi, T.; Yamaki, T.; Itoh, H.; Asai, K. Band Gap Narrowing of Titanium Dioxide by Sulfur Doping. Appl. Phys. Lett. 2002, 81, 454-456.

(14) Varley, J. B.; Janotti, A.; Van de Walle, C. G. Mechanism of Visible-Light Photocatalysis in Nitrogen-Doped $\mathrm{TiO}_{2}$. Adv. Mater. 2011, 23, 2343-2347.

(15) Li, H.; Li, J.; Huo, Y. Highly Active $\mathrm{TiO}_{2} \mathrm{~N}$ Photocatalysts Prepared by Treating $\mathrm{TiO}_{2}$ Precursors in $\mathrm{NH}_{3} /$ Ethanol Fluid under Supercritical Conditions. J. Phys. Chem. B 2006, 110, 1559-1565.

(16) Sakai, Y. W.; Obata, K.; Hashimoto, K.; Irie, H. Enhancement of Visible Light-Induced Hydrophilicity on Nitrogen and Sulfur-Codoped $\mathrm{TiO}_{2}$ Thin Films. Vacuum 2008, 83, 683-687.

(17) Umezawa, N.; Janotti, A.; Rinke, P.; Chikyow, T.; Van de Walle, C. G. Optimizing Optical Absorption of $\mathrm{TiO}_{2}$ by Alloying with $\mathrm{TiS}_{2}$. Appl. Phys. Lett. 2008, 92, 041104/1-041104/3.

(18) Long, R.; English, N. J. Synergistic Effects of Bi/S Codoping on Visible Light-Activated Anatase $\mathrm{TiO}_{2}$ Photocatalysts from First Principles. J. Phys. Chem. C 2009, 113, 8373-8377.

(19) Wang, P.; Liu, Z.; Lin, F.; Zhou, G.; Wu, J.; Duan, W.; Gu, B.-L.; Zhang, S. B. Optimizing Photoelectrochemical Properties of $\mathrm{TiO}_{2}$ by Chemical Codoping. Phys. Rev. B 2010, 82, 193103/1-193103/4.

(20) Chen, X.; Liu, L.; Yu, P. Y.; Mao, S. S. Increasing Solar Absorption for Photocatalysis with Black Hydrogenated Titanium Dioxide Nanocrystals. Science 2011, 331, 746-750.

(21) Hu, Y. H. A Highly Efficient Photocatalyst-Hydrogenated Black $\mathrm{TiO}_{2}$ for the Photocatalytic Splitting of Water. Angew. Chem., Int. Ed. 2012, 51, 12410-12412.

(22) Ho, W.; Yu, J. C.; Lee, S. Low-Temperature Hydrothermal Synthesis of S-Doped $\mathrm{TiO}_{2}$ with Visible Light Photocatalytic Activity. J. Solid State Chem. 2006, 179, 1171-1176.

(23) Li, H.; Zhang, X.; Huo, Y.; Zhu, J. Supercritical Preparation of a Highly Active S-Doped $\mathrm{TiO}_{2}$ Photocatalyst for Methylene Blue Mineralization. Environ. Sci. Technol. 2007, 41, 4410-4414.

(24) Rockafellow, E. M.; Stewart, L. K.; Jenks, W. S. Is Sulfur-Doped $\mathrm{TiO}_{2}$ an Effective Visible Light Photocatalyst for Remediation? Appl. Catal., B 2009, 91, 554-562.

(25) Onida, G.; Reining, L.; Rubio, A. Electronic Excitations: Density-Functional versus Many-Body Green's-Function Approaches. Rev. Mod. Phys. 2002, 74, 601-659.

(26) Kohn, W.; Sham, L. J. Self-Consistent Equations Including Exchange and Correlation Effects. Phys. Rev. 1965, 140, A1133A1138.

(27) Ceperley, D. M.; Alder, B. J. Ground State of the Electron Gas by a Stochastic Method. Phys. Rev. Lett. 1980, 45, 566-569.

(28) Perdew, J. P.; Zunger, A. Self-Interaction Correction to DensityFunctional Approximations for Many-Electron Systems. Phys. Rev. B 1981, 23, 5048-5079.

(29) Kresse, G.; Joubert, D. From Ultrasoft Pseudopotentials to the Projector Augmented-Wave Method. Phys. Rev. B 1999, 59, 17581775 .

(30) Kresse, G.; Furthmüller, J. Efficient Iterative Schemes for Ab Initio Total-Energy Calculations Using a Plane-Wave Basis Set. Phys. Rev. B 1996, 54, 11169-11186.

(31) Kresse, G.; Furthmüller, J. Efficiency of Ab-Initio Total Energy Calculations for Metals and Semiconductors Using a Plane-Wave Basis Set. Comput. Mater. Sci. 1996, 6, 15-50.

(32) Gajdoš, M.; Hummer, K.; Kresse, G.; Furthmüller, J.; Bechstedt, F. Linear Optical Properties in the Projector-Augmented Wave Methodology. Phys. Rev. B 2006, 73, 045112/1-045112/9.

(33) Schleife, A.; Varley, J. B.; Fuchs, F.; Rödl, C.; Bechstedt, F.; Rinke, P.; Janotti, A.; Van de Walle, C. G. Tin Dioxide from First Principles: Quasiparticle Electronic States and Optical Properties. Phys. Rev. B 2011, 83, 035116/1-035116/9.

(34) Schleife, A.; Rödl, C.; Furthmüller, J.; Bechstedt, F. Electronic and Optical Properties of $\mathrm{Mg}_{x} \mathrm{Zn}_{1-x} \mathrm{O}$ and $\mathrm{Cd}_{x} \mathrm{Zn}_{1-x} \mathrm{O}$ from $\mathrm{Ab}$ Initio Calculations. New J. Phys. 2011, 13, 085012/1-085012/24.
(35) Rödl, C.; Fuchs, F.; Furthmüller, J.; Bechstedt, F. Ab Initio Theory of Excitons and Optical Properties for Spin-Polarized Systems: Application to Antiferromagnetic MnO. Phys. Rev. B 2008, 77, 184408/1-184408/12.

(36) Hedin, L. New Method for Calculating the One-Particle Green's Function with Application to the Electron-Gas Problem. Phys. Rev. 1965, 139, A796-A823.

(37) Fuchs, F.; Rödl, C.; Schleife, A.; Bechstedt, F. Efficient $O\left(N^{2}\right)$ Approach to Solve the Bethe-Salpeter Equation for Excitonic Bound States. Phys. Rev. B 2008, 78, 085103/1-085103/13.

(38) Schmidt, W. G.; Glutsch, S.; Hahn, P. H.; Bechstedt, F. Efficient $O\left(N^{2}\right)$ Method to Solve the Bethe-Salpeter Equation. Phys. Rev. B 2003, 67, 085307/1-085307/7.

(39) (a) Schleife, A. Exciting Imperfection: Real-Structure Effects in Magnesium-, Cadmium-, and Zinc-Oxide. Ph.D. Thesis, FriedrichSchiller-Universität, Jena, Germany, 2010. (b) Schleife, A. Electronic and Optical Properties of $\mathrm{MgO}, \mathrm{ZnO}$, and $\mathrm{CdO}$; Südwestdeutscher Verlag für Hochschulschriften: Saarbrücken, Germany, 2011. 